\title{
Three-Dimensional Microstructural Characterization of Novel Chalcogenide Nanocomposites for Gradient Refractive Index Applications
}

\author{
Hugues Francois-Saint-Cyr ${ }^{1 \dagger *}$, Myungkoo Kang ${ }^{2 \dagger}$, Isabelle Martin ${ }^{1 \dagger}$, Stoichko Antonov ${ }^{3}$, Ty J. Prosa ${ }^{1}$, \\ and Kathleen Richardson ${ }^{2}$ \\ 1. CAMECA Instruments Inc., 5470 Nobel Drive, Madison, WI 53711 USA. \\ 2. CREOL, the College of Optics and Photonics, University of Central Florida, Orlando, FL 32816 USA. \\ 3. University Science and Technology Beijing, 30 Xueyuan Rd, Haidian District, Beijing, 100083 China. \\ $\dagger$ These authors contributed to this work equally. \\ * Corresponding author: hugues.fsc@ametek.com
}

Infrared components based on spherical lenses have been widely used in imaging systems [1]. However, their chromatic aberration leads to an increase in the number, volume, and weight of optical components, an inconvenience counterbalanced by the use of gradient refractive index (GRIN) materials [1]. One of strategies to form GRIN profiles within a medium involves spatial control of conversion from a base glass to a glass-ceramic. Phase separation seen in $\mathrm{GeSe}_{2}-\mathrm{As}_{2} \mathrm{Se}_{3}-\mathrm{PbSe}$ chalcogenide bulk glasses has gained interest since the Pb-rich amorphous phases, once thermally treated, can be exclusively converted into nanocrystals with an index greater than that of the Pb-deficient matrix which remains amorphous $[2,3]$. Since the number density and the crystallinity of the Pb-rich phases can be spatially controlled, this material system has been considered a promising technique to create a GRIN profile within a single component [Figs. 1(a) and 1(b)]. It is vital to have experimental approaches which allow us to identify the distribution and type of the co-existing phases in a 3-dimensional (3-D) space since the connectivity has a significant impact on the scattering of electromagnetic waves going through the nanocomposite. Here, we report a progress on a parallel comparison of TEM and APT analyses to reveal true distribution and types of co-existing phases within the glasses for GRIN applications.

$20 \mathrm{GeSe}_{2}-60 \mathrm{As}_{2} \mathrm{Se}_{3}-20 \mathrm{PbSe}$ bulk glasses were prepared using conventional melt-quenching technique [2]. The microstructure and composition of the glasses were investigated using both a FEI Tecnai F30 TEM and CAMECA LEAP 5000XR. Fig. 1(c) shows a bright field TEM image collected from the glass where dark particles with an average diameter of $\sim 30 \mathrm{~nm}$ are distributed in a bright matrix. The contrast in brightness between the particles and the matrix indicates that the atomic percentage of heavy elements in the particles is greater than that in the matrix. Fig. 1(d) shows composition profiles of constituents including $\mathrm{Se}, \mathrm{Pb}$, As, and Ge extracted from the white dotted line on the region in Fig. 1(c) where Pb, the heaviest element in the glass, is segregated in the dark particles. Meanwhile, since regions analyzable using TEM must be electron-transparent and therefore very thin, the technique provides a nanocomposite's microstructure from a 2-D perspective and has a limitation in identifying how the two co-existing phases are distributed in 3-D space. Meanwhile, the atom-by-atom field evaporation process, induced by needle-shape specimens of about 100-nm radius or less at the apex, lead to a 3D reconstruction of atoms from these glasses. At the nanoscale, this technique enables identification of the distribution of inclusions within their matrix, often not possible in projection methods such as TEM where those inclusions overlap each other [4].

The preparation of the glass into a needle shape with the FIB is challenging due to its unusually fast ion milling behavior. This issue was overcome by capping the polished bulk material with a sacrificial $60 \mathrm{~nm}$ Ni layer prior to the standard FIB-based specimen preparation procedure and provided uniformity and 
reproducibility after the final low-kV clean up to remove $\mathrm{Ga}^{+}$implantation. The best data quality and survivability was found at low base temperature $(30 \mathrm{~K})$, high detection rate $(2 \%)$, low pulse frequency $(50 \mathrm{kHz})$, and extremely low laser pulse energy (LPE) (10 - 100fJ). Despite background levels on the order of $50-100 \mathrm{ppm} / \mathrm{nsec}$, excellent mass resolving power $(>1000)$ of the mass spectrum allowed identification of several Pb-based and (As-Se)-based compounds, as shown in Fig. 1(e). APT ion maps for Pb-rich regions and AsSe-rich regions were anti-correlated, corroborating the findings provided by the line scans in the TEM [5].

\section{References:}

[1] J. Teichman et al., Gradient Index Optics at DARPA, The Institute for Defense Analyses (2014).

[2] L. Sisken, PhD Dissertation, University of Central Florida (2017).

[3] M. Kang et al., Adv. Mater. 30 (2018), 1803628.

[4] D. J. Larson et al., "Local Electrode Atom Probe Tomography”, Springer, New York (2013).

[5] The authors acknowledge the partial support of UCF's Pre-eminent Post-doctoral Scholar Program.

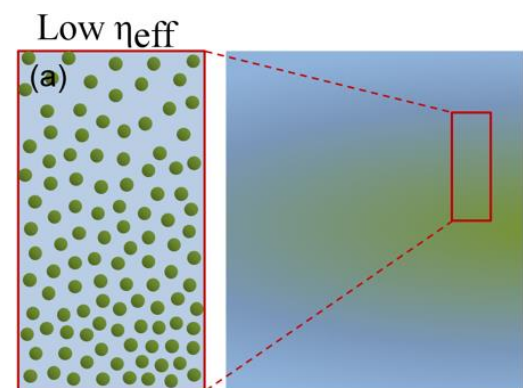

High $\eta_{\text {eff }}$
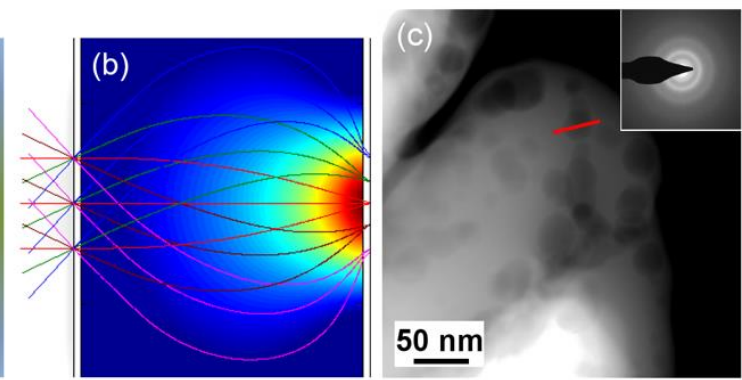

(e)

$$
\begin{array}{crrr} 
& & \mathrm{GeSe}_{2}^{1+} \mathbf{P b S e}_{2}^{2+} \\
\mathrm{As}_{2}^{1+} & \mathrm{AsSS}_{4}^{2+} & \mathrm{As}_{3} \mathrm{Se}_{3}^{2+} & \mathbf{P b S e}^{1+} \\
\mathrm{Ge}_{2} \mathrm{Se}_{2}^{2+} & \mathrm{As}_{3} \mathrm{Se}_{2}^{2+} & \mathrm{AsSe}_{2}^{1+} & \mathrm{GeAs}_{2} \mathrm{Se}_{4}^{2+}
\end{array}
$$

$$
\begin{array}{lll}
\mathrm{As}_{3}^{2+} & \mathrm{Ge}_{2} \mathrm{Se}_{2}^{2+} & \mathrm{As}_{3} \mathrm{Se}_{2}^{2+}
\end{array}
$$

$\mathrm{As}_{2} \mathrm{Se}^{1+} \mathrm{As}_{3} \mathrm{Se}_{4}^{2+}$

$\mathrm{PbSe}^{1+}$

$\mathrm{GeSe}_{3}^{1+}$
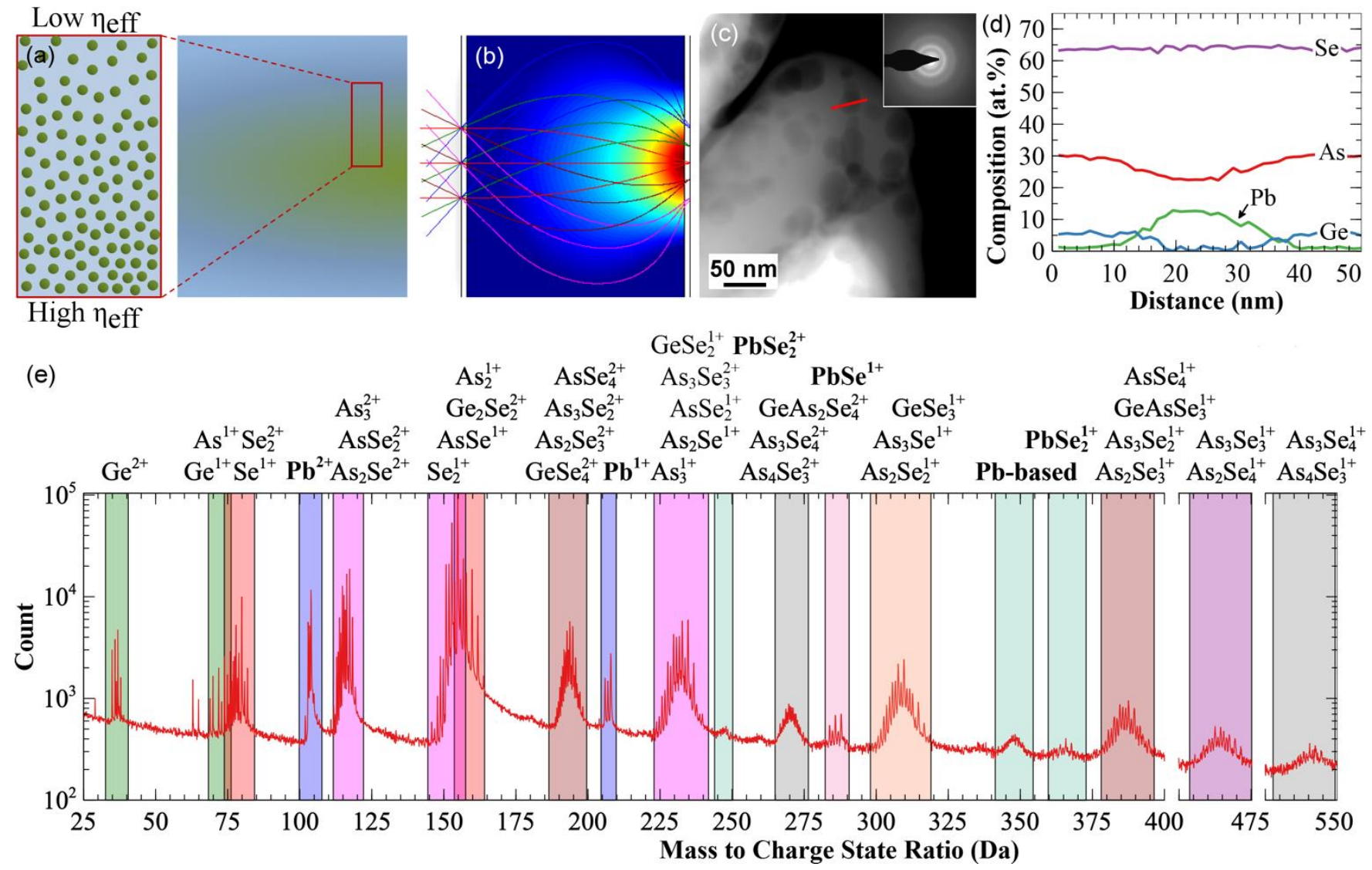
$\mathrm{As}_{4} \mathrm{Se}_{3}^{2+}$ $\mathrm{As}_{2} \mathrm{Se}_{2}^{1+}$

$\mathrm{AsSe}_{4}^{1+}$

$\mathrm{GeAsSe}_{3}^{1+}$

Figure 1. (a) a GRIN medium where the density of high index nanocrystals (green) are spatially varied within a low refractive matrix (blue). (b) a flat lens based on a GRIN profile. (c) TEM data collected from a powder specimen of the glass. (d) composition profiles of $\mathrm{Se}, \mathrm{Pb}, \mathrm{As}$, and $\mathrm{Ge}$ extracted from the red solid line in (c). (e) a mass spectrum collected from an APT tip of the glass where peaks corresponding to its elemental constituents and their compounds are seen. Pb-based compounds are indicated in bold. 